\title{
Acetylsalicylic Acid Compared with Enoxaparin for the Prevention of Thrombosis and Mechanical Ventilation in COVID-19 Patients: A Retrospective Cohort Study
}

\author{
Heba Wagih Abdelwahab ${ }^{1}$. Shaker Wagih Shaltout ${ }^{2} \cdot$ Hazem A. Sayed Ahmed $^{3}$. Ahmed Mahmoud Fouad ${ }^{4}$. \\ Eric Merrell ${ }^{5}$. Jeffrey B. Riley ${ }^{6} \cdot$ Rasha Salama $^{4} \cdot$ Ahmed Gharib Abdelrahman $^{3}$. Edward Darling ${ }^{6} \cdot$ Ghada Fadel $^{11}$. \\ Mohamed S. A. Elfar ${ }^{7} \cdot$ Khaled Sabry $^{8} \cdot$ Jaffer Shah $^{9}$ (D) Hossam Amin ${ }^{10} \cdot$ Gary F. Nieman $^{11} \cdot$ Adel Mishriky $^{4}$ \\ Hani Aiash ${ }^{3,6,11}$
}

Accepted: 6 July 2021 / Published online: 30 July 2021

(c) The Author(s), under exclusive licence to Springer Nature Switzerland AG 2021

\begin{abstract}
Background and Objective Low-dose acetylsalicylic acid (ASA, aspirin) is a well-known and frequently studied drug for primary and secondary prevention of disease due to its anti-inflammatory and coagulopathic effects. COVID-19 complications are attributed to the role of thrombo-inflammation. Studies regarding the use of low-dose ASA in COVID-19 are limited. For this reason, we propose that the use of low-dose ASA may have protective effects in COVID-19-related thromboembolism and lung injury. This study was conducted to assess the efficacy of low-dose ASA compared with enoxaparin, an anticoagulant, for the prevention of thrombosis and mechanical ventilation.

Methods We conducted a retrospective cohort study on COVID-19-confirmed hospitalized patients at the Mansoura University Quarantine Hospital, outpatients, and home-isolated patients from September to December 2020 in Mansoura governorate, Egypt. Binary logistic regression analysis was used to assess the effect of ASA compared with enoxaparin on thromboembolism, and mechanical ventilation needs.

Results This study included 225 COVID-19 patients. Use of ASA-only (81-162 mg orally daily) was significantly associated with reduced thromboembolism (OR 0.163, $p=0.020$ ), but both low-dose ASA and enoxaparin, and enoxaparin-only $(0.5 \mathrm{mg} / \mathrm{kg}$ subcutaneously (SC) daily as prophylactic dose or $1 \mathrm{mg} / \mathrm{kg} \mathrm{SC}$ every 12 hours as therapeutic dose) were more protective (odds ratio [OR] 0.010, OR 0.071, respectively, $p<0.001$ ). Neither ASA-only nor enoxaparin-only were associated with a reduction in mechanical ventilation needs. Concomitant use of low-dose ASA and enoxaparin was associated with reduced mechanical ventilation (OR 0.032, 95\% CI 0.004-0.226, $p=0.001$ ).

Conclusions Low-dose ASA-only use may reduce the incidence of COVID-19-associated thromboembolism, but the reduction may be less than that of enoxaparin-only, and both ASA and enoxaparin. Concomitant use of ASA and enoxaparin demonstrates promising results with regard to the reduction of thrombotic events, and mechanical ventilation needs.
\end{abstract}

\section{Introduction}

The COVID-19 pandemic has had a devastating impact globally. COVID-19 manifests with a wide spectrum of clinical presentations including asymptomatic (40-45\%) [1] or symptomatic, with symptomatic cases further classified

Heba Wagih Abdelwahab, Shaker Wagih Shaltout and Hazem A. Sayed Ahmed are joint first authors.

Jaffer Shah

jaffer.shah@kateb.edu.af

Extended author information available on the last page of the article as mild (40\%), moderate (40\%), severe (15\%), and critical (5\%). Patients with severe or critical illness develop complications such as acute respiratory distress syndrome (ARDS), septic shock, thromboembolism, and/or multiple organ dysfunction [2]. The pathogenesis of these complications is poorly characterized but thought to be due to a combination of severe inflammation, platelets activation, endotheliopathy, and coagulopathy [3-6].

Thromboembolic events are frequently observed in COVID-19 patients (16-31\%) and are likely most common in the moderate-to-severe cases that are often encountered in the hospitalized setting [7-9]. One study noted a high 


\section{Key Points}

Among COVID-19 patients, thromboembolic events were less likely to occur among patients on low-dose acetylsalicylic acid alone, but both acetylsalicylic acid and enoxaparin, and enoxaparin alone were more effective in reducing these events.

Use of acetylsalicylic acid alone and enoxaparin alone was not associated with a reduction in mechanical ventilation needs.

Concomitant use of low-dose aspirin and enoxaparin was associated with reduced mechanical ventilation.

incidence of venous thromboembolic (VTE) complications in hospitalized COVID-19 patients (26\%), with $12 \%$ of patients having pulmonary embolism (PE) with or without deep venous thrombosis (DVT) and $14 \%$ with DVT alone [10]. Little is known about the incidence of thrombosis in the outpatient setting especially for those with mild disease. Reports have described precipitous embolic events in mild disease managed in the outpatient setting necessitating emergent hospitalization [11]. COVID-19 patients may be predisposed to both arterial and or venous thrombosis [12]. In a recent study, the incidence of arterial thrombosis was observed to be about twice that of VTE in hospitalized COVID-19 patients (11.1\% vs 6.2\%) [9].

Low-dose acetylsalicylic acid (ASA, aspirin) may have a putative role in targeting the pathogenesis of COVID-19 complications through its anti-inflammatory, antiplatelet aggregation, anticoagulant effects, and pleiotropic effects on endothelial function [13,14], as well as its antiviral activity against RNA viruses in the respiratory tract [15]. Low-dose ASA is recommended for secondary prevention of arterial thrombosis in COVID-19 [16, 17], further studies described a possible role in primary prevention of arterial thrombosis as well $[11,14,18]$. Use of ASA in COVID-19 patients prior to hospitalization may have an important role in prevention of ARDS and mortality [19]. Prior to COVID era, a meta-analysis concluded that antiplatelet therapies such as ASA were associated with a lower incidence of ARDS and reduced mortality in critically ill patients [20].

A recent retrospective cohort study found that ASA use in hospitalized COVID-19 patients was associated with decreased risk of mechanical ventilation, intensive care unit (ICU) admission, and in-hospital mortality [21]. A propensity-matched retrospective study revealed that in-hospital ASA compared to no antiplatelet therapy was associated with a significantly reduced cumulative incidence of inhospital mortality among hospitalized adult patients with
COVID-19 (hazard ratio 0.522 [0.336-0.812]) [22]. A recent meta-analysis concluded that low-dose ASA use during or prior to hospitalization was associated with a significant reduction in mortality among patients with COVID-19 [23]. Yuan et al found that prehospital ASA use was not associated with decreased mortality in hospitalized COVID-19 patients with coronary artery disease [24]. Another recent meta-analysis supported this finding [25].

Data regarding the protective effects of ASA therapy on arterial thrombosis, and risk of mechanical ventilation are limited. This study aims to assess the effectiveness of lowdose ASA compared with enoxaparin (ENX) for the prevention of thrombotic events, and mechanical ventilation need.

\section{Methods}

\subsection{Study Design and Patients}

We conducted a retrospective cohort observational study between September and December 2020. Ethical approval was obtained from the Institutional Review Board (IRB) at Mansoura University (code number: R.20.08.971).

Convenience sampling was used on 234 COVID-19 patients, who were aged at least 18 years, and had a confirmed SARS-CoV-2 infection by the qualitative real-time polymerase chain reaction (RT-PCR) of a nasal swab. Nine patients were excluded because they were pregnant, had a history of end-stage renal disease with coagulopathy, and had inadequate laboratory and/or radiological data. Consequently, 225 COVID-19 patients were included in this study.

\subsection{Data, Definitions, and the Perceived Treatment}

Demographics, comorbidities, laboratory, and radiological data of hospitalized patients were aretrieved from the medical records at Mansoura University's Quarantine Hospital, Mansoura governorate, Egypt. Similar data were retrieved, as complete as possible, from home-isolated patients and patients who preferred outpatient follow-up. Radiological data included chest computed tomography (CT) findings (e.g. ground glass opacity [GGO], crazy-paving pattern, pulmonary consolidation, fibrosis, sub-pleural lines, and the halo sign), which were defined according to the standard glossary for thoracic imaging reported by the Fleischner Society [26]. A semi-quantitative CT severity scoring proposed by Pan et al was calculated per each of the 5 lobes considering the extent of anatomic involvement, as follows: 0 : no involvement; $1:<5 \%$ involvement; $2: 5-25 \%$ involvement; 3: 26-50\% involvement; 4: 51-75\% involvement; 5: > $75 \%$ involvement. Summation of each individual lobar score resulted in a global CT score (0-25) [27]. 
COVID-19 cases were classified according to WHO definition and are defined as follows: moderate COVID19 defined as the presence of clinical signs of pneumonia (fever, cough, dyspnea, fast breathing) but no signs of severe pneumonia, including oxygen saturation by pulse oxymetry $\left(\mathrm{SpO}_{2}\right) \geq 90 \%$ on room air, severe COVID-19 defined by the presence of oxygen saturation $<90 \%$ on room air or respiratory rate $>30$ breaths per minute in adults or presence of signs of severe respiratory distress, i.e., accessory muscle use, inability to complete full sentences; critical COVID-19 defined as the presence of ARDS, sepsis, septic shock or the need for vasopressor therapy or non-invasive or invasive mechanical ventilation [2].

Patients were categorized as control group (who do not receive ASA, others antiplatelet agents, ENX, or others anticoagulant), ASA alone, ENX alone, or both ASA and ENX (ASA-ENX). ASA group included COVID-19 patients who were released for home isolation or presented with a primary thromboembolic event and were not on an anticoagulant. Patients with concomitant antiplatelet therapies were excluded from ASA group.

Use of ASA was defined as administration of low-dose ASA (81-162 mg orally [PO] daily) for other underlying conditions within 7 days prior to-or within $24 \mathrm{~h}$ of COVID19 diagnosis. This definition was based on the rapid onset $(0-4 \mathrm{~h})$ and the prolonged duration of action of ASA when chewed or swallowed. The irreversible platelet-inhibitory effects of ASA can last for the life span of human platelets (7-10 days) $[28,29]$.

Enoxaparin, an injectable low-molecule weight heparin (LMWH), was the only received anticoagulant in our sample. Enoxaparin intake was defined as receiving ENX for other underlying conditions prior to hospital admission, or after the diagnosis of COVID-19. According to the Egyptian protocol of COVID-19 management, prophylactic dose enoxaparin sodium $(0.5 \mathrm{mg} / \mathrm{kg}$ subcutaneously [SC] daily) was administrated for hospitalized patients with moderate COVID-19 and having D-Dimer 500-1000 ng/mL. Therapeutic dose (1 mg/kg SC every 12 hours) was administrated for hospitalized patients with moderate COVID-19 and having D-Dimer more than $1000 \mathrm{ng} \mathrm{mL}$ or hospitalized patients with severe or critical COVID-19. Thus, enoxaparin was not prescribed to non-hospitalized patients [30].

In our study, patients with moderate COVID-19 received oral hydroxychloroquine (400 mg twice per day on first day then $200 \mathrm{mg}$ twice per day for 6 days) plus oral ivermectin (36 mg on day [0-3-6]). while moderate COVID19 patients with a high risk and arterial oxygen saturation $\left(\mathrm{SaO}_{2}\right)<92 \%$ received remdesivir $(200 \mathrm{mg}$ intravenously [IV] on Day 1 followed by $100 \mathrm{mg}$ IV daily for 5 days). Remdesivir was also prescribed for patients with severe or critical COVID-19. Steroids (dexamethasone $6 \mathrm{mg}$ IV or its oral equivalent) were prescribed for patients with moderate COVID-19 who had severe dyspnea, a respiratory rate more than 24 breaths/min or their CT showed rapid deterioration, while steroids (dexamethasone $6 \mathrm{mg}$ IV or methylprednisolone $1 \mathrm{mg} / \mathrm{kg} / 24 \mathrm{~h}$ ) were administrated for those patients with severe or critical COVID-19 [30].

\subsection{Outcomes}

The primary outcome was the occurrence of thrombotic events, defined prior to implementing this study as DVT, pulmonary embolism, peripheral arterial occlusion, ischemic stroke, ST-elevation myocardial infarction, or bowel ischemia. In our study, thromboembolic events were arterial events. Cardiologists diagnosed COVID-19 cases with coronary artery disease using echocardiogram and cardiac enzymes, while neurologists diagnosed stroke in COVID-19 patients using CT brain scan.

Another study outcome showed the need for mechanical ventilation following a diagnosis of COVID-19. Indications of invasive mechanical ventilation in our study were: failed non-invasive ventilation, or not available or not practical, partial pressure of oxygen $\left(\mathrm{PO}_{2}\right)<60 \mathrm{mmHg}$ despite oxygen supplementation, progressive hypercapnia, respiratory acidosis $(\mathrm{PH}<7.30)$, progressive or refractory septic shock, disturbed consciousness level (Glascow coma score $\leq 8$ ) or deterioration of consciousness level from baseline [30].

\subsection{Statistical Analysis}

All statistical analyses were performed using IBM SPSS Statistics for Windows, version 25 (IBM Corp., Armonk, NY, USA). Categorical variables were presented as number and percentage of patients and were compared between groups using the Chi-squared or Fisher's exact tests. Continuous variables were presented as mean \pm standard deviation (SD), and tested for normality with the Kolmogorov-Smirnov test. Nonparametric continuous variables were compared among groups using the Kruskal Wallis Test and the $p$ values were adjusted for multiple comparisons using Bonferroni Correction. Binary logistic regression models were fitted to determine the adjusted associations between ASA use and outcomes (i.e. thrombotic events, and the need for mechanical ventilation) after controlling for confounders. Selection of confounders was guided by the significance level in bivariate associations, collinearity, the change in model's $R$-square, and in published literature recommendations. Selected confounders included patient's age, previous comorbidities such as diabetes, hypertension, established cardiovascular diseases (CVD), and COVID-19 severity. $p$ values $<0.05$ were considered statistically significant. 


\section{Results}

This study included 225 patients with a confirmed diagnosis of COVID-19. Patients on ASA, ENX, or both were significantly older than patients not on these medications $(p<$ 0.001 ), while there was no statistically significant difference between sexes. Diabetes mellitus, hypertension, and stroke were the most frequent comorbidities observed amongst patients receiving both ASA and ENX, while cardiac and coronary artery diseases were most frequent among patient receiving ASA alone. The majority of patients on either ENX alone or both ASA and ENX had severe COVID-19 (79.7\% and $88.6 \%$, respectively), while $64.5 \%$ of patients on ASA alone had moderate COVID-19 ( $p<0.001)$ (Table 1).

Thrombo-embolic events included cerebrovascular stroke and myocardial infarction. Cerebrovascular stroke represented the majority of events in the control, ASA alone, and ASA-ENX groups $(63.6 \%, 87.5 \%$, and $100 \%$, respectively), while myocardial infarction constituted $66.7 \%$ of all thrombo-embolic events in ENX alone group.

Radiological findings showed that patients on either ENX alone or both ASA and ENX had higher severity scores than patients on ASA alone or controls $(p=0.015)$. No statistically significant differences existed among the study groups regarding diagnostic laboratory findings with the exception of platelets count, alanine aminotransferase (ALT) and aspartate aminotransferase (AST) (Table 2).

In multivariate analysis, thrombo-embolic events were significantly less likely to occur among patients on both ASA and ENX, ENX alone, or ASA alone when compared to control patients (OR 0.010, OR 0.071, and OR 0.163, respectively, $p<0.05)$ when adjusted for age, diabetes mellitus, hypertension, established CVD, and COVID-19 severity (Table 3). The need for mechanical ventilation, adjusted for patient's age, diabetes mellitus, hypertension, established CVD, and COVID-19 severity, was less likely for patients on both ASA and ENX compared with control patients (OR $0.032,95 \%$ CI $0.004-0.226, p=0.001)$, Table 4 .

\section{Discussion}

This retrospective cohort study found that the use of lowdose ASA alone, ENX alone, and both low-dose ASA and ENX was associated with a lower risk of thrombotic events. COVID-19 severity, the presence of established CVD, and increased age were the positive predictors of the development of these events. Concomitant use of ASA and ENX was associated with reduced mechanical ventilation need. The positive predictors of mechanical ventilation needs were COVID-19 severity, the presence of hypertension, and increased age.
In our study, vascular thrombotic events were significantly more frequent among control patients and patients on ASA alone compared with patients on ENX alone or both ASA and ENX. This finding in these non-hospitalized patients with moderate-to-severe COVID-19 is troubling as the risk for thrombosis is not limited exclusively to hospital settings.

Control patients had insignificant elevations of D-Dimer levels, C-reactive protein (CRP), and ferritin, which may reflect hypercoagulopathy and hyperinflammatory state. The pathogenesis of thrombi formation in these patients involves an evolution of Virchow's triad: stasis from microthrombi, hyperviscosity, and immobility related to malaise; abnormal coagulability likely due to immune-mediated factors; and virus-associated endothelial damage. Individuals in the ASA alone group had comorbidities such as established cardiovascular diseases, diabetes mellitus and hypertension, which may outweigh the protective effect of ASA for CVD, promote disease escalation and abnormal coagulation and leave many patients vulnerable to escalation of illness severity.

All thrombotic events in our study were arterial thrombosis. Use of ENX or low-dose ASA in combination with ENX, may have contributed to the absence of venous thrombosis in our current study. The incidence rates of arterial thrombosis among control patients $(30.6 \%)$, and patients on ASA alone (25.8\%) or ENX alone (17.1\%) were higher than the incidence rates described in previous studies $[9,31]$. This finding may be related to our small sample size within each group. Prior studies found varied incidence rates of arterial thrombosis. A systematic review found that arterial thrombosis occurred in $4.4 \%$ of critically ill COVID19 patients [31], while another meta-analysis described a higher incidence of thromboembolic events in hospitalized COVID-19 patients (7.2-40.8\%) with a predominance of venous thromboembolic events [32]. Bilaloglu et al found that $11.1 \%$ of 3334 hospitalized COVID-19 patients developed arterial thrombosis [9].

Our study supports the use of low-dose ASA, ENX, and ASA plus ENX to reduce the risk of arterial thrombosis in COVID-19 patients; however, randomized control trials are needed to assess the causality effects of these therapies. These findings reflected the protective effect of low-dose ASA and ENX therapy on thrombosis in COVID-19 patients likely due to a combination of antithrombotic, anti-inflammatory, and antiviral effects in COVID-19 patients [33-35]. Administration of an anticoagulant is still recommended for hospitalized patients with COVID-19 unless there are other indications in non-hospitalized patients with COVID-19 [17].

Despite the effectiveness of ASA on thrombosis prevention in this study, ASA plus ENX therapy was more effective than ASA alone. This is possibly due to a synergistic or additive effect between these therapies. However, in our study 
Table 1 Demographic and clinical characteristics, and outcomes of the study groups $(\mathrm{N}=225)$

\begin{tabular}{|c|c|c|c|c|c|}
\hline Variable & Control $(n=36)$ & ASA alone $(n=31)$ & ENX alone $(n=123)$ & ASA-ENX $(n=35)$ & $p$-value \\
\hline Age $(y)$, mean \pm SD (range) & $\begin{array}{l}44 \pm 16.5 \\
(20-75)\end{array}$ & $\begin{array}{l}56 \pm 16.1^{* * *} \\
(25-85)\end{array}$ & $\begin{array}{l}58 \pm 14.7^{* * *} \\
(25-90)\end{array}$ & $\begin{array}{l}61 \pm 14.3^{* *} \\
(33-88)\end{array}$ & $<0.001^{*}$ \\
\hline \multicolumn{6}{|l|}{ Age category (y) } \\
\hline$<45$ & $16(44.4 \%)$ & $9(29.0 \%)$ & $24(19.5 \%)$ & $6(17.1 \%)$ & \multirow[t]{3}{*}{$0.018^{*}$} \\
\hline $45-65$ & $17(47.2 \%)$ & $12(38.7 \%)$ & $58(47.2 \%)$ & $19(54.3 \%)$ & \\
\hline$>65$ & $3(8.3 \%)$ & $10(32.3 \%)$ & $41(33.3 \%)$ & $10(28.6 \%)$ & \\
\hline \multicolumn{6}{|l|}{ Patient sex } \\
\hline Female & $24(66.7 \%)$ & $15(48.4 \%)$ & $66(53.7 \%)$ & $18(51.4 \%)$ & \multirow[t]{2}{*}{0.426} \\
\hline Male & $12(33.3 \%)$ & $16(51.6 \%)$ & $57(46.3 \%)$ & $17(48.6 \%)$ & \\
\hline \multicolumn{6}{|l|}{ Comorbidities } \\
\hline None & $24(66.7 \%)$ & $9(29.0 \%)$ & $44(35.8 \%)$ & $6(17.1 \%)$ & NA \\
\hline Diabetes mellitus & $4(11.1 \%)$ & $12(38.7 \%)$ & $53(43.1 \%)$ & $17(48.6 \%)$ & $0.003^{*}$ \\
\hline Hypertension & $5(13.9 \%)$ & $15(48.4 \%)$ & $56(45.5 \%)$ & $22(62.9 \%)$ & $<0.001^{*}$ \\
\hline Asthma & $2(5.6 \%)$ & $1(3.2 \%)$ & $6(4.9 \%)$ & $3(8.6 \%)$ & 0.766 \\
\hline Cardiac/coronary artery disease & 0 & $8(25.8 \%)$ & $4(3.3 \%)$ & $8(22.9 \%)$ & $<0.001^{*}$ \\
\hline Stroke & $2(5.6 \%)$ & $4(12.9 \%)$ & $4(3.3 \%)$ & $6(17.1 \%)$ & $0.014^{*}$ \\
\hline Gastrointestinal symptoms & $2(5.6 \%)$ & $8(25.8 \%)$ & $26(21.1 \%)$ & $11(32.4 \%)$ & $0.041^{*}$ \\
\hline \multicolumn{6}{|l|}{ COVID-19 severity (on-diagnosis) } \\
\hline Moderate & $29(80.6 \%)$ & $20(64.5 \%)$ & $25(20.3 \%)$ & $4(11.4 \%)$ & \multirow[t]{2}{*}{$<0.001^{*}$} \\
\hline Severe/critical & $7(19.4 \%)$ & $11(35.5 \%)$ & $98(79.7 \%)$ & $31(88.6 \%)$ & \\
\hline $\begin{array}{l}\mathrm{SPO}_{2} \% \text { (on-admission), mean } \pm \mathrm{SD} \\
\quad \text { (range) }\end{array}$ & $92.30 \pm 9.1(59-99)$ & $91.9 \pm 4.7(80-98)$ & $82.7 \pm 12.1(48-98)^{* * *}$ & $85.2 \pm 8.6(62-97)^{* * *}$ & $<0.001^{*}$ \\
\hline \multicolumn{6}{|l|}{ Ventilatory support (on-diagnosis) } \\
\hline Room-air & $25(69.4 \%)$ & $13(41.9 \%)$ & $21(17.1 \%)$ & $4(11.4 \%)$ & \multirow[t]{4}{*}{$<0.001^{*}$} \\
\hline Oxygen therapy & $10(27.8 \%)$ & $16(51.6 \%)$ & $80(65.0 \%)$ & $30(85.7 \%)$ & \\
\hline Continuous positive airway pressure & $1(2.8 \%)$ & $2(6.5 \%)$ & $11(8.9 \%)$ & $1(2.9 \%)$ & \\
\hline Invasive mechanical ventilation & 0 & 0 & $11(8.9 \%)$ & 0 & \\
\hline \multicolumn{6}{|l|}{ Outcomes } \\
\hline Thromboembolic events & $11(30.6 \%)$ & $8(25.8 \%)$ & $21(17.1 \%)$ & $2(5.9 \%)$ & $0.042^{*}$ \\
\hline Need for mechanical ventilation & $6(16.7 \%)$ & $11(35.5 \%)$ & $49(39.8 \%)$ & $7(20.0 \%)$ & $0.021^{*}$ \\
\hline
\end{tabular}

$N A$ not applicable, $A S A$ acetylsalicylic acid, $A S A-E N X$ both acetylsalicylic acid and enoxaparin, $E N X$ enoxaparin, $\mathrm{Sp} \mathrm{O}_{2}$ oxygen saturation

*Statistically significant $p$ value (<0.05); Kruskal Wallis Test for age and $\mathrm{SPO}_{2} \%$; Chi-square or Fisher's for other categorical variables

${ }^{* *}$ Significantly different from the control group ( $p$ value adjusted for multiple comparisons with Bonferroni Correction)

**** Significantly different from the ASA group ( $p$ value adjusted for multiple comparisons with Bonferroni Correction)

we could not differentiate between the effect of low-dose ASA use for primary and secondary cardiovascular disease during pre-hospitalization or prior to home-isolation because of the small number of patients on low-dose ASA therapy for secondary prevention (26 patients). Chow et al [21] and Sahai et al [36] did not find an association between low-dose ASA use and prevention of thrombotic events; this finding is related to the lower percentage of reported thrombosis events in their sample.

We encourage the community to consider primary prophylaxis of thrombotic complications in individuals who may already be at an increased risk for thrombosis and test positive for COVID-19 in the outpatient setting. Our focus as a community, at times, seems to be on the treatment of those most critically ill, often neglecting those who fall in limbo between recovery and hospitalization. For this, we recommend the community consider the use of ASA for prevention of arterial and venous thrombotic events. With proper risk-stratification, the use of outpatient thromboprophylaxis may provide patients with a low-risk opportunity to defend against advanced disease while targeted therapy and vaccine production has time to be properly developed to be effective therapy.

According to our results, low-dose ASA was not effective in reducing the need for mechanical ventilation. In contrast, Chow et al found that low-dose ASA use was associated with reduced mechanical ventilation need [21]. Patients taking ASA in their study had less oxygen support on hospital 
Table 2 Radiological and laboratory findings on diagnosis among the study groups $(\mathrm{N}=225)$

\begin{tabular}{|c|c|c|c|c|c|}
\hline Findings & Control $(n=36)$ & ASA alone $(n=31)$ & ENX alone $(n=123)$ & ASA-ENX $(n=35)$ & $p$ value \\
\hline $\begin{array}{l}\text { Total Lung Severity Score }(n= \\
76), \text { mean } \pm \text { SD (range) }\end{array}$ & $\begin{array}{l}10.9 \pm 5.5 \\
(0-19)\end{array}$ & $\begin{array}{l}9.7 \pm 4.0 \\
(2-15)\end{array}$ & $\begin{array}{l}14.7 \pm 4.9^{a} \\
(5-25)\end{array}$ & $\begin{array}{l}15.3 \pm 4.3 \\
(9-20)\end{array}$ & $0.015^{*}$ \\
\hline CT Phenotype $(N=193)$ & $(n=18)$ & $(n=28)$ & $(n=113)$ & $(n=34)$ & \\
\hline Type L & $10(55.6 \%)$ & $15(53.6 \%)$ & $42(37.2 \%)$ & $17(50.0 \%)$ & 0.2 \\
\hline Type H & $8(44.4 \%)$ & $13(46.4 \%)$ & $71(62.8 \%)$ & $17(50.0 \%)$ & \\
\hline Hemoglobin (g/dL) & $12.2 \pm 1.8$ & $11.5 \pm 1.6$ & $11.8 \pm 1.9$ & $11.6 \pm 1.9$ & 0.365 \\
\hline White blood cells $(\times 1000)$ & $9.1 \pm 7.4$ & $8.6 \pm 4.0$ & $9.0 \pm 4.5$ & $9.2 \pm 4.2$ & 0.409 \\
\hline Lymphocytes (\%) & $25.9 \pm 14.3$ & $23.5 \pm 16.3$ & $19.7 \pm 11.3$ & $17.3 \pm 13.4$ & 0.327 \\
\hline Platelets $(\times 1000)$ & $235.7 \pm 110.4$ & $210.3 \pm 63.4$ & $198.2 \pm 80.3^{* *}$ & $194.1 \pm 63.5$ & $0.045^{*}$ \\
\hline D-Dimer (ng/mL) & $2300 \pm 2700$ & $1300 \pm 1400$ & $1200 \pm 2000$ & $500 \pm 700$ & 0.556 \\
\hline Ferritin $(\mathrm{ng} / \mathrm{mL})$ & $451.6 \pm 696.9$ & $151.0 \pm 146.1$ & $228.4 \pm 186.4$ & $147.5 \pm 96.9$ & 0.846 \\
\hline C-reactive protein $(\mathrm{mg} / \mathrm{L})$ & $63.6 \pm 72.9$ & $71.6 \pm 53.5$ & $48.4 \pm 41.7$ & $43.5 \pm 31.6$ & 0.377 \\
\hline $\begin{array}{l}\text { Erythrocyte sedimentation rate } \\
(\mathrm{mm} / \text { hour })\end{array}$ & $31.7 \pm 23.9$ & $36.3 \pm 31.2$ & $37.4 \pm 18.7$ & $41.7 \pm 40.2$ & 0.873 \\
\hline Albumin $(\mathrm{g} / \mathrm{dL})$ & $3.7 \pm 0.8$ & $3.3 \pm 0.5$ & $3.4 \pm 0.6$ & $3.5 \pm 0.5$ & 0.304 \\
\hline Alanine aminotransferase (U/L) & $24.6 \pm 12.0$ & $31.8 \pm 17.3$ & $46.8 \pm 42.4^{* *}$ & $33.8 \pm 17.3$ & $0.001 *$ \\
\hline Aspartate aminotransferase (U/L) & $25.5 \pm 14.9$ & $38.9 \pm 20.1^{* *}$ & $53.5 \pm 47.9^{* *}$ & $32.9 \pm 15.7$ & $<0.001^{*}$ \\
\hline Bilirubin (mg/dL) & $1.0 \pm 1.1$ & $0.7 \pm 0.2$ & $0.8 \pm 0.6$ & $0.8 \pm 0.2$ & 0.752 \\
\hline Serum creatinine $(\mathrm{mg} / \mathrm{dL})$ & $1.5 \pm 1.9$ & $1.5 \pm 0.9$ & $1.5 \pm 2.7$ & $1.2 \pm 0.4$ & 0.346 \\
\hline
\end{tabular}

ASA acetylsalicylic acid, ASA-ENX both acetylsalicylic acid and enoxaparin, CT computed tomography, ENX enoxaparin

*Statistically significant $p$ value $(<0.05)$; Kruskal Wallis Test and Fisher's exact test

**Significantly different from the control group ( $p$ value adjusted for multiple comparisons with Bonferroni Correction)

${ }^{a}$ Significantly different from the aspirin group ( $p$ value adjusted for multiple comparisons with Bonferroni Correction)

Table 3 Logistic regression analysis for predicting thromboembolic events in the study groups $(N=225)$

\begin{tabular}{|c|c|c|c|}
\hline \multirow[t]{2}{*}{ Variables } & \multicolumn{2}{|c|}{ Adjusted model $^{\mathrm{a}}$} & \multirow[t]{2}{*}{$p$ value } \\
\hline & OR & $95 \% \mathrm{CI}$ & \\
\hline Constant & 0.052 & & $<0.001^{*}$ \\
\hline Age $(y)$ & 1.034 & $1.000-1.069$ & $0.047 *$ \\
\hline Severe/critical COVID-19 (vs moderate) & 6.008 & $1.570-22.99$ & $0.009 *$ \\
\hline \multicolumn{4}{|l|}{ Comorbidities } \\
\hline Diabetes mellitus (vs No) & 0.867 & $0.367-2.051$ & 0.746 \\
\hline Hypertension (vs No) & 1.740 & $0.704-4.301$ & 0.230 \\
\hline Established cardiovascular disease (vs No) & 3.289 & $1.057-10.23$ & $0.040^{*}$ \\
\hline \multicolumn{4}{|l|}{ Study groups (vs non-ASA and non-ENX) } \\
\hline Acetylsalicylic acid alone & 0.163 & $0.035-0.752$ & $0.020 *$ \\
\hline Enoxaparin alone & 0.071 & $0.018-0.280$ & $<0.001 *$ \\
\hline Both acetylsalicylic acid and enoxaparin & 0.010 & $0.001-0.078$ & $<0.001 *$ \\
\hline
\end{tabular}

Variables entered on step 1: Age (y), COVID-19 Severity (severe/critical vs moderate), diabetes mellitus (Y/N), hypertension (Y/N), established cardiovascular disease (Y/N), and study groups (vs non-acetylsalicylic acid and non-anticoagulant)

$A S A$ acetylsalicylic acid, $C I$ confidence interval, ENX enoxaparin, $O R$ odds ratio

*Statistically significant $p$ value $(<0.05)$

${ }^{a}$ Binary Logistic Regression Model: Hosmer and Lemeshow $\chi^{2}(d f)=5.926(8), p=0.655$; Negelkerke $R$ Square $=0.310$; Overall correct classification $=83.9 \%$

admission and lower fibrinogen values. This could represent less severe illness and inflammatory states, which could conceivably produce a more favorable outcome [37].
Nevertheless, our study demonstrated that low-dose ASA plus ENX was associated with decreased mechanical ventilation needs. This reflects the protective and desirable effect 
Table 4 Logistic regression analysis for predicting the need for mechanical ventilation in the study groups $(N=225)$

\begin{tabular}{|c|c|c|c|}
\hline \multirow[t]{2}{*}{ Variables } & \multicolumn{2}{|c|}{ Adjusted model $^{\mathrm{a}}$} & \multirow[t]{2}{*}{$p$ value } \\
\hline & OR & $95 \% \mathrm{CI}$ & \\
\hline Constant & 0.002 & & $<0.001^{*}$ \\
\hline Age (years) & 1.053 & $1.019-1.088$ & $0.002^{*}$ \\
\hline Severe/critical COVID-19 (vs moderate) & 69.00 & $10.43-456.7$ & $<0.001^{*}$ \\
\hline \multicolumn{4}{|l|}{ Comorbidities } \\
\hline Diabetes mellitus (vs No) & 0.718 & $0.326-1.580$ & 0.410 \\
\hline Hypertension (vs No) & 2.414 & $1.066-5.466$ & $0.035^{*}$ \\
\hline Established cardiovascular disease (vs No) & 3.179 & $0.903-11.19$ & 0.072 \\
\hline \multicolumn{4}{|l|}{ Study groups (vs non-ASA and non-ENX) } \\
\hline Acetylsalicylic acid alone & 1.095 & $0.130-8.791$ & 0.932 \\
\hline Enoxaparin alone & 0.276 & $0.051-1.391$ & 0.125 \\
\hline Both acetylsalicylic acid and enoxaparin & 0.032 & $0.004-0.226$ & $0.001^{*}$ \\
\hline
\end{tabular}

Variables entered on step 1: Age (y), COVID-19 Severity (severe/critical vs moderate), diabetes mellitus (Y/N), hypertension (Y/N), established cardiovascular disease (Y/N), and study groups (vs non-acetylsalicylic acid and non-anticoagulant)

$A S A$ acetylsalicylic acid, $C I$ confidence interval, $E N X$ enoxaparin, $O R$ odds ratio

* Statistically significant $p$ value $(<0.05)$

${ }^{a}$ Binary Logistic Regression Model: Hosmer and Lemeshow $\chi^{2}(d f)=6.969(8), p=0.540$; Negelkerke $R$ Square $=0.541$; Overall correct classification $=79.1 \%$

of both ASA and ENX. A finding that enoxaparin alone was not associated with decreased rate of mechanical ventilation is incongruent with a previous study regarding reduced mechanical ventilation with therapeutic anticoagulant, which may be related to the limited sample size of ENX-only group in our study [38].

Enoxaparin was used in this study was due to provider preference and COVID-19 treatment protocols at the participating institution. Results from this study, while specific to enoxaparin, likely extend to other LMWHs (i.e. dalteparin), unfractionated heparin (UFH) and newer direct oral anticoagulants (DOACs) (i.e. betrixaban, rivaroxaban and apixaban) based on the proposed protective mechanism of thromboprophylaxis and the absence of known other unique therapeutic characteristics in COVID-19. Enoxaparin, dalteparin and UFH have shown comparable efficacy in the prevention of DVT in traumatic injuries [39]; however, while dalteparin was found to be non-superior to UFH in the prevention of DVT in critically ill patients, there was a noted reduction in the event of pulmonary embolus in the PROTECT trial [40].

Comparatively, between ENX and dalteparin, studies have not shown a difference in the prevention of DVT and PE for trauma patients [41]. Lastly, LMWH and DOACs have been thoroughly studied in acutely ill medical patients. The APEX trial reported no statistical difference in the efficacy of betrixaban versus ENX for the prevention of VTE [42], the Magellan trial reported a reduction in VTE in the rivaroxaban group when compared to ENX [43], and the ADOPT trial showed that apixaban was non-superior to
ENX in the prevention VTE [44]. More studies are required to evaluate the efficacy of different anticoagulant agents in the prevention of VTE related to COVID-19.

To our knowledge, this is the first Egyptian study to assess the efficacy of low-dose ASA compared with ENX in the prevention of COVID-19-associated thrombosis, and mechanical ventilation.

This study has some limitations. Its observational design lacking randomization cannot establish causality. This study also suffered from a limited sample size, and single institutional analysis, so its result cannot be generalized. With the small number of participants on low-dose ASA, the effect of ASA use on primary and secondary atherosclerotic cardiovascular disease (ASCVD) could not be determined separately. Other confounders such as smoking, body mass index, and use of drugs for ASCVD prevention, especially statin, and other antiplatelet agents were not included in hospitalized patients. Lastly, the risk of bleeding associated with the use of ASA, ENX and concomitant ASA and ENX therapy was not assessed.

\section{Conclusion}

Use of low-dose ASA alone is significantly associated with a lower risk of COVID-19-related thromboembolism, but ENX alone, and both low-dose ASA and ENX were more effective than ASA in the reduction of thromboembolism. Concomitant ASA and ENX therapy are associated with a reduction in the need for mechanical ventilation. Further 
randomized control trials are needed to establish potential causal relationships.

Acknowledgements Deepest appreciation for the COVID-19 patients who participated in this study.

\section{Declarations}

Funding No financial support was obtained from any individual, institutions, agencies, drug industries or organizations.

Competing interests The authors declare that they have no competing interests.

Ethics approval This study was approved by the Mansoura Institutional Research Board Ethics (code number: R.20.08.971), which waived the need for informed consent.

Consent to participate The Ethics Committee of Mansoura University waived the need for informed consent from the study population for the collection, analysis, and publication of the retrospectively obtained and anonymized data for this non-interventional study.

Consent to publish Not applicable.

Code availability Not applicable.

Availability of data and materials The datasets used and/or analyzed during the current study are available from the corresponding author on reasonable request.

Author contributions HWA, SWS, and HASA contributed equally to this work and share first authorship. HA put the idea of the research, built the research team, designed the study, revised the manuscript, and approved the final version of manuscript. HWA and SWS participated in designing the study, collected the data, revised the manuscript, and approved the final version of manuscript. HASA participated in designing the study, wrote the manuscript draft, and approved the final version of manuscript. AMF participated in designing the study, analyzed the data, revised the manuscript, and approved the final version of manuscript. AM and AGA participated in designing the study and in statistical analysis, revised the manuscript, and approved the final version. EM, ED, JBR, GF, and GFN participated in designing this study parallel to another IRB in USA, revised this manuscript, and approved the final version. RS, ME-f, KS, JS, and HA participated in designing the study, revised the manuscript, and approved the final version.

\section{References}

1. Oran DP, Topol EJ. Prevalence of asymptomatic SARS-CoV-2 infection: a narrative review. Ann Intern Med. 2020;173(5):362-7. https://doi.org/10.7326/M20-3012.

2. World Health Organization. Clinical management of COVID19: interim guidance, 27 May 2020. World Health Organization. License: CC BY-NC-SA 3.0 IGO. https://www.who.int/publicatio ns/i/item/clinical-management-of-covid-19. Accessed 8 Dec 2020.

3. Zeng F, Huang Y, Guo Y, Yin M, Chen X, Xiao L, et al. Association of inflammatory markers with the severity of COVID-19: a meta-analysis. Int J Infect Dis. 2020;96:467-74. https://doi.org/ 10.1016/j.ijid.2020.05.055.

4. Varga Z, Flammer AJ, Steiger P, Haberecker M, Andermatt R, Zinkernagel AS, et al. Endothelial cell infection and endotheliitis in COVID-19. Lancet. 2020;395(10234):1417-8. https://doi.org/ 10.1016/S0140-6736(20)30937-5.

5. Ackermann M, Verleden SE, Kuehnel M, Haverich A, Welte T, Laenger F, et al. Pulmonary vascular endothelialitis, thrombosis, and angiogenesis in Covid-19. N Engl J Med. 2020;383(2):120-8. https://doi.org/10.1056/NEJMoa2015432.

6. Goshua G, Pine AB, Meizlish ML, Chang CH, Zhang H, Bahel $\mathrm{P}$, et al. Endotheliopathy in COVID-19-associated coagulopathy: evidence from a single-centre, cross-sectional study. Lancet Haematol. 2020;7(8):e575-82. https://doi.org/10.1016/S23523026(20)30216-7.

7. Lodigiani C, Iapichino G, Carenzo L, Cecconi M, Ferrazzi P, Sebastian T, et al. Venous and arterial thromboembolic complications in COVID-19 patients admitted to an academic hospital in Milan, Italy. Thromb Res. 2020;191:9-14. https://doi.org/10. 1016/j.thromres.2020.04.024.

8. Klok FA, Kruip MJHA, van der Meer NJM, Arbous MS, Gommers DAMPJ, Kant KM, et al. Incidence of thrombotic complications in critically ill ICU patients with COVID-19. Thromb Res. 2020;191:145-7. https://doi.org/10.1016/j.thromres.2020.04.013.

9. Bilaloglu S, Aphinyanaphongs Y, Jones S, Iturrate E, Hochman J, Berger JS. Thrombosis in hospitalized patients with COVID-19 in a New York City health system. JAMA. 2020;324(8):799-801. https://doi.org/10.1001/jama.2020.13372.

10. Porfidia A, Valeriani E, Pola R, Porreca E, Rutjes AWS, Di Nisio M. Venous thromboembolism in patients with COVID-19: systematic review and meta-analysis. Thromb Res. 2020;196:67-74. https://doi.org/10.1016/j.thromres.2020.08.020.

11. Shawkat A, Merrell ET, Fadel GA, Amzuta I, Amin H, Shah AJ, et al. Multiple thrombotic events in a 67-year-old man 2 weeks after testing positive for SARS-CoV-2: a case report. Am J Case Rep. 2020;21: e925786. https://doi.org/10.12659/AJCR.925786.

12. Bikdeli B, Madhavan MV, Jimenez D, Chuich T, Dreyfus I, Driggin E, et al. COVID-19 and thrombotic or thromboembolic disease: implications for prevention, antithrombotic therapy, and follow-up: JACC state-of-the-art review. J Am Coll Cardiol. 2020;75(23):2950-73. https://doi.org/10.1016/j.jacc.2020.04.031.

13. Dzeshka MS, Shantsila A, Lip GY. Effects of aspirin on endothelial function and hypertension. Curr Hypertens Rep. 2016;18(11):83. https://doi.org/10.1007/s11906-016-0688-8.

14. Sayed Ahmed HA, Merrell E, Ismail M, Joudeh AI, Riley JB, Shawkat A, et al. Rationales and uncertainties for aspirin use in COVID-19: a narrative review. Fam Med Com Health. 2021;9(2): e000741. https://doi.org/10.1136/fmch-2020-000741.

15. Glatthaar-Saalmüller B, Mair KH, Saalmüller A. Antiviral activity of aspirin against RNA viruses of the respiratory tract-an in vitro study. Influenza Other Respir Viruses. 2017;11(1):85-92. https:// doi.org/10.1111/irv.12421.

16. The European Society for Cardiology. ESC Guidance for the Diagnosis and Management of CV Disease during the COVID-19 Pandemic. https://www.escardio.org/static-file/Escardio/EducationGeneral/Topic\%20pages/Covid-19/ESC\%20Guidance\%20Doc ument/ESC-Guidance-COVID-19-Pandemic.pdf. Accessed 5 Mar 2021.

17. COVID-19 Treatment Guidelines Panel. Coronavirus Disease 2019 (COVID-19) Treatment Guidelines. National Institutes of Health. https://files.covid19treatmentguidelines.nih.gov/guide lines/covid19treatmentguidelines.pdf. Accessed 8 Dec 2020.

18. Griffin DO, Jensen A, Khan M, Chin J, Chin K, Parnell R, Awwad C, Patel D. Arterial thromboembolic complications in COVID19 in low-risk patients despite prophylaxis. Br J Haematol. 2020;190(1):e11-3. https://doi.org/10.1111/bjh.16792.

19. Zhou X, Li Y, Yang Q. Antiplatelet therapy after percutaneous coronary intervention in patients with COVID-19: implications from clinical features to pathologic findings. Circulation. 
2020;141(22):1736-8. https://doi.org/10.1161/CIRCULATIO NAHA.120.046988.

20. Wang L, Li H, Gu X, Wang Z, Liu S, Chen L. Effect of antiplatelet therapy on acute respiratory distress syndrome and mortality in critically ill patients: a meta-analysis. PLoS ONE. 2016;11(5): e0154754. https://doi.org/10.1371/journal.pone.0154754.

21. Chow JH, Khanna AK, Kethireddy S, Yamane D, Levine A, Jackson AM, et al. Aspirin use is associated with decreased mechanical ventilation, ICU admission, and in-hospital mortality in hospitalized patients with COVID-19. Anesth Analg. 2021;132(4):930-41. https://doi.org/10.1213/ANE.0000000000 005292.

22. Meizlish ML, Goshua G, Liu Y, Fine R, Amin K, Chang E, et al. Intermediate-dose anticoagulation, aspirin, and in-hospital mortality in COVID-19: a propensity score-matched analysis. Am J Hematol. 2021;96(4):471-9. https://doi.org/10.1002/ajh.26102.

23. Martha JW, Pranata R, Lim MA, Wibowo A, Akbar MR. Active prescription of low-dose aspirin during or prior to hospitalization and mortality in COVID-19: a systematic review and meta-analysis of adjusted effect estimates. Int J Infect Dis. 2021;108:6-12. https://doi.org/10.1016/j.ijid.2021.05.016.

24. Yuan S, Chen P, Li H, Chen C, Wang F, Wang DW. Mortality and pre-hospitalization use of low-dose aspirin in COVID-19 patients with coronary artery disease. J Cell Mol Med. 2020;25(2):126373. https://doi.org/10.1111/jcmm.16198.

25. Salah HM, Mehta JL. Meta-analysis of the effect of aspirin on mortality in COVID-19. Am J Cardiol. 2021;142:158-9. https:// doi.org/10.1016/j.amjcard.2020.12.073.

26. Hansell DM, Bankier AA, MacMahon H, McLoud TC, Müller NL, Remy J. Fleischner Society: glossary of terms for thoracic imaging. Radiology. 2008;246(3):697-722. https://doi.org/10. 1148/radiol.2462070712.

27. Pan F, Ye T, Sun P, Gui S, Liang B, Li L, et al. Time course of lung changes at chest CT during recovery from coronavirus disease 2019 (COVID-19). Radiology. 2020;295(3):715-21. https:// doi.org/10.1148/radiol.2020200370.

28. Masoud HH, Elassal, G, Hassany M, Shawky A, Abdel Hakim M, Zaky, S, et al. Management Protocol for COVID-19 Patients MoHP Protocol for COVID19 November 2020. https://www.resea rchgate.net/publication/345813633_Management_Protocol_for_ COVID-19_Patients_MoHP_Protocol_for_COVID19_Novem ber_2020/link/5faeb3df92851cf24cca8643/download. Accessed 9 Mar 2021.

29. Eikelboom JW, Hirsh J, Spencer FA, Baglin TP, Weitz JI. Antiplatelet drugs: antithrombotic therapy and prevention of thrombosis, 9th ed: American College of Chest Physicians Evidence-Based Clinical Practice Guidelines. Chest. 2012;141(2):e89S-e119S. https://doi.org/10.1378/chest.11-2293.

30. Cho J. A paradigm shift in platelet transfusion therapy. Blood. 2015;125(23):3523-5. https://doi.org/10.1182/ blood-2015-04-640649.

31. Cheruiyot I, Kipkorir V, Ngure B, Misiani M, Munguti J, Ogeng'o J. Arterial thrombosis in coronavirus disease 2019 patients: a rapid systematic review. Ann Vasc Surg. 2021;70:273-81. https:// doi.org/10.1016/j.avsg.2020.08.087.

32. Kunutsor SK, Laukkanen JA. Incidence of venous and arterial thromboembolic complications in COVID-19: a systematic review and meta-analysis. Thromb Res. 2020;196:27-30. https://doi.org/ 10.1016/j.thromres.2020.08.022.

33. Diaz T, Trachtenberg BH, Abraham SJK, KosagiSharaf R, DurantArchibold AA. Aspirin bioactivity for prevention of cardiovascular injury in COVID-19. Front Cardiovasc Med. 2020;7: 562708. https://doi.org/10.3389/fcvm.2020.562708.

34. Liu J, Li J, Arnold K, Pawlinski R, Key NS. Using heparin molecules to manage COVID-2019. Res Pract Thromb Haemost. 2020;4(4):518-23. https://doi.org/10.1002/rth2.12353.

35. Godino C, Scotti A, Maugeri N, Mancini N, Fominskiy E, Margonato A, et al. Antithrombotic therapy in patients with COVID19?-rationale and evidence. Int J Cardiol. 2021;324:261-6. https://doi.org/10.1016/j.ijcard.2020.09.064.

36. Sahai A, Bhandari R, Godwin M, McIntyre T, Chung MK, Iskandar JP, et al. Effect of aspirin on short-term outcomes in hospitalized patients with COVID-19. Vasc Med. 2021. https:// doi.org/10.1177/1358863X211012754.

37. Giorgi-Pierfranceschi M. Is aspirin effective in preventing ICU admission in patients with COVID-19 pneumonia? Anesth Analg. 2021;132(5):e89-90. https://doi.org/10.1213/ANE.0000000000 005401.

38. Roomi SS, Saddique M, Ullah W, Haq S, Ashfaq A, Madara J, et al. Anticoagulation in COVID-19: a single-center retrospective study. J Community Hosp Intern Med Perspect. 2021;11(1):1722. https://doi.org/10.1080/20009666.2020.1835297.

39. Miano TA, Cuker A, Christie JD, Martin N, Smith B, Makley AT, et al. Comparative effectiveness of enoxaparin vs dalteparin for thromboprophylaxis after traumatic injury. Chest. 2018;153(1):133-42. https://doi.org/10.1016/j.chest.2017.08.008.

40. PROTECT Investigators for the Canadian Critical Care Trials Group and the Australian and New Zealand Intensive Care Society Clinical Trials Group, Cook D, Meade M, Guyatt G, Walter S, Heels-Ansdell D, et al. Dalteparin versus unfractionated heparin in critically ill patients. N Engl J Med. 2011;364(14):1305-14. https://doi.org/10.1056/NEJMoa1014475.

41. Okoye OT, Gelbard R, Inaba K, Esparza M, Belzberg H, Talving $\mathrm{P}$, Teixeira PG, et al. Dalteparin versus enoxaparin for the prevention of venous thromboembolic events in trauma patients. Eur J Trauma Emerg Surg. 2014;40(2):183-9. https://doi.org/10.1007/ s00068-013-0333-z.

42. Cohen AT, Harrington RA, Goldhaber SZ, Hull RD, Wiens BL, Gold A, Hernandez AF, Gibson CM, APEX Investigators. Extended thromboprophylaxis with betrixaban in acutely ill medical patients. N Engl J Med. 2016;375(6):534-44. https://doi.org/ 10.1056/NEJMoa1601747.

43. Cohen AT, Spiro TE, Büller HR, Haskell L, Hu D, Hull R, Mebazaa A, Merli G, Schellong S, Spyropoulos AC, Tapson V, MAGELLAN Investigators. Rivaroxaban for thromboprophylaxis in acutely ill medical patients. N Engl J Med. 2013;368(6):51323. https://doi.org/10.1056/NEJMoa1111096.

44. Goldhaber SZ, Leizorovicz A, Kakkar AK, Haas SK, Merli G, Knabb RM, Weitz JI, ADOPT Trial Investigators. Apixaban versus enoxaparin for thromboprophylaxis in medically ill patients. N Engl J Med. 2011;365(23):2167-77. https://doi.org/10.1056/ NEJMoa1110899. 


\section{Authors and Affiliations}

Heba Wagih Abdelwahab ${ }^{1}$. Shaker Wagih Shaltout ${ }^{2} \cdot$ Hazem A. Sayed Ahmed $^{3} \cdot$ Ahmed Mahmoud Fouad $^{4}$. Eric Merrell ${ }^{5}$. Jeffrey B. Riley ${ }^{6} \cdot$ Rasha Salama $^{4} \cdot$ Ahmed Gharib Abdelrahman $^{3}$. Edward Darling ${ }^{6} \cdot$ Ghada Fadel $^{11}$. Mohamed S. A. Elfar ${ }^{7} \cdot$ Khaled Sabry $^{8} \cdot$ Jaffer Shah ${ }^{9}$ (I) Hossam Amin ${ }^{10} \cdot$ Gary F. Nieman $^{11} \cdot$ Adel Mishriky ${ }^{4}$ Hani Aia $\mathrm{sh}^{3,6,11}$

1 Department of Chest Medicine, Faculty of Medicine, Mansoura University, Mansoura, Egypt

2 Department of Tropical Medicine, Faculty of Medicine, Port Said University, Port Fuad, Egypt

3 Department of Family Medicine, Faculty of Medicine, Suez Canal University, Ismailia, Egypt

4 Department of Public Health, Occupational and Environmental Medicine, Faculty of Medicine, Suez Canal University, Ismailia, Egypt

5 Department of Medicine, State University of New York Upstate Medical University, Syracuse, NY, USA

6 Department of Cardiovascular Perfusion, State University of New York Upstate Medical University, Syracuse, NY, USA
7 Touro College of Osteopathic Medicine, New York, NY, USA

8 Department of Orthopedic Surgery, Faculty of Medicine, Port Said University, Port Fuad, Egypt

9 Medical Research Center, Kateb University, Kabul, Afghanistan

10 Department of Critical Care, New York Medical College, Valhalla, NY, USA

11 Department of Surgery, State University of New York Upstate Medical University, Syracuse, NY, USA 